\title{
Recent extensions of the INCL4 model for spallation reactions
}

\author{
Thierry Aoust ${ }^{\mathrm{a}, \mathrm{b}}$, Alain Boudard ${ }^{\mathrm{c}}$, Joseph Cugnon ${ }^{\mathrm{a}, *}$, Jean-Christophe David ${ }^{\mathrm{c}}$, \\ Pierre Henrotte ${ }^{\mathrm{a}}$, Sylvie Leray ${ }^{\mathrm{c}}$, Claude Volant ${ }^{\mathrm{c}}$ \\ ${ }^{a}$ University of Liège, allée du 6 Août 17, bât. B5, B-4000 Liège 1, Belgium \\ ${ }^{\mathrm{b}} \mathrm{SCK}-\mathrm{CEN}$, Boeretang 200, B-2400 Mol, Belgium \\ ${ }^{\mathrm{c}} D A P N I A / S P h N, C E A$-Saclay, F-91191 Gif-sur-Yvette Cedex, France
}

Available online 3 March 2006

\begin{abstract}
New improvements of the INCL4 cascade model, bearing on cluster production, nucleon and pion potentials and Pauli blocking at low energy, are reviewed.

(C) 2006 Elsevier B.V. All rights reserved.

PACS: 25.40.Sc; 25.80.Hp; 28.90. +i
\end{abstract}

Keywords: Spallation reactions; Intranuclear cascade; INCL4

\section{Introduction}

The Liège intranuclear cascade model, INCL4, has been shown to give, when coupled with the Schmidt evaporation-fission code [1,2], a fairly good description of a large amount of data concerning proton-induced spallation reactions in the $200 \mathrm{MeV}-2 \mathrm{GeV}$ range [3-9]. Remarkably enough, this was achieved without any fitting of parameters. On the other hand, the model is based on a few assumptions. Some of these are either solidly founded or are at the premises of the model, such as the assumption of well-ordered (in space and time) binary collisions. Questioning the latter amounts to question the model itself. Some other assumptions may appear more prone to a critical evaluation (e.g. the treatment of the $\Delta$ degrees of freedom) or may even look dubious. As an example, the assumption of independent nucleons, though largely supported by our knowledge of the nucleus, precludes the emission of clusters. In addition, the INCL4 model still suffers of few, but definite, limitations. The most important one, concerning the energy range, comes from the fact that this model has been developed mainly in the frame of the

\footnotetext{
${ }^{*}$ Corresponding author. Tel.: + 3243663601 ; fax: +3243663672 .

E-mail address: cugnon@plasma.theo.phys.ulg.ac.be (J. Cugnon).
}

European HINDAS project [10], wherein it was assigned to deal with the above-mentioned energy range.

Here, we want to report on several improvements and extensions of the model pions, and on some improvements at low energy. In this paper, we are more interested in discussing the physics aspects of the modifications rather than in a thorough confrontation with the data.

\section{Improvements}

\subsection{Cluster formation in the cascade stage}

We have implemented a relatively simple model in INCL4, which allows the production of clusters in the cascade stage. When a nucleon arrives at the surface and is going to be emitted, it is checked whether it can drag along with it one or several nucleons, forming a cluster. Such an emission is allowed if nucleons are sufficiently close to each other in phase space. The following clusters $\left(\mathrm{d}, \mathrm{t},{ }^{3} \mathrm{He}\right.$ and ${ }^{4} \mathrm{He}$ ) are considered up to now, but the method could be extended to heavier clusters. When a large cluster has been built, it is emitted provided the energy of the cluster is sufficiently large and provided it succeeds the test for transmission through the relevant Coulomb barrier. If not, the smaller clusters inside the original one are tested for emission, and so on. If no cluster can be emitted, the 
Table 1

Multiplicities per primary reaction obtained in proton-induced reactions on ${ }^{208} \mathrm{~Pb}$ at $1.2 \mathrm{GeV}$

\begin{tabular}{lccl}
\hline & Without clusters & With clusters & Exp \\
\hline$n$, (casc) & 4.13 & 3.52 & \\
$n$, (tot) & 14.61 & 14.27 & \\
$n$, (free + bound) & 15.93 & 16.52 & \\
$n, E>20 \mathrm{MeV}$ & 3.17 & 2.67 & $2.7 \pm 0.3$ \\
\hline
\end{tabular}

Experimental data are from Ref. [11].

original nucleon is simply emitted. We refer to Ref. [13] for more detail. In simple words, this model is a surface coalescence model based on the dynamic phase space distribution in the surface region at any time. It is different from the usual coalescence model: it allows emission at any moment and, as a matter of fact, it does violate the scaling laws of the coalescence model.

A satisfactory agreement with experimental results is obtained, as shown in Ref. [13]. It is interesting to look at multiplicities (see Table 1). The production of clusters in the cascade stage reduces the number of free neutrons emitted in this stage, as expected. Evaporation is not really affected. However the total number of neutrons (free or hidden inside clusters) is enhanced. The reason is that it is energetically easier to extract nucleons bound in clusters than free nucleons. The number of neutrons of more than $20 \mathrm{MeV}$ is slightly reduced, which improves our previous calculations.

We do not expect this simple model to work at lower energy than, say, $100 \mathrm{MeV}$, where more subtle production mechanisms are presumably at work, but we did not check this point yet.

\subsection{The nuclear mean field}

In contrast with most of INC models, we have introduced a nuclear potential well which depends upon the isospin $\left(T_{3}\right)$ and the energy $(E)$ of the nucleons, in accordance with the phenomenology of the optical-model potential [12]. Roughly speaking, the depth of the potential is linearly decreasing with the nucleon energy until the latter reaches $E_{0} \sim 200 \mathrm{MeV}$, beyond which it basically vanishes $(i=n, p)$ :

$$
\begin{aligned}
V_{0}^{i}(E) & =V_{0}^{i}-\alpha_{i}\left(E-E_{F}^{i}\right) \text { for } E<E_{0} \\
& =0 \text { for } E>E_{0} .
\end{aligned}
$$

Furthermore, inside the nucleus, $V_{0}^{n}$ is larger than $V_{0}^{p}$ and $E_{F}^{n} \approx E_{F}^{p}$. The consequences of introducing these phenomenological potentials are discussed extensively in Ref. [14]. The main effect is due to the isospin-dependence: less neutrons and more protons are emitted in the cascade stage (see Table 2). This is due to the average binding energy, which has increased for neutrons and decreased for protons. It is also interesting to note that the neutron
Table 2

Multiplicities per primary reaction obtained in proton-induced reactions on ${ }^{208} \mathrm{~Pb}$ at $800 \mathrm{MeV}$

\begin{tabular}{lllll}
\hline Standard & $T_{3}$ & $T_{3}$ & $E$ & Exp \\
\hline$n, E>20 \mathrm{MeV}$ & 2.48 & 2.28 & 2.21 & $1.9 \pm 0.2$ \\
$n, E>2 \mathrm{MeV}$ & 9.30 & 9.26 & 9.23 & $10.4 \pm 1.4$ \\
$p, E>20 \mathrm{MeV}$ & 2.07 & 2.20 & 2.18 & \\
$p, E>2 \mathrm{MeV}$ & 2.55 & 2.70 & 2.65 & \\
\hline
\end{tabular}

Comparison of the results for various types of nuclear mean field: standard (2nd column), isospin-dependent (3rd column), isospin- and energy-dependent (4th column). Experimental data are from Ref. [11].

quasi-elastic peak has shifted toward larger energy loss, by about $20 \mathrm{MeV}$, coming closer to the experimental results. The proton quasi-elastic peak remains basically unshifted.

\subsection{The pion mean field}

Introducing the pion potential in a simulation is not a trivial point. Identifying the latter with the real part of the optical potential, as for the nucleons, is not indicated, since this quantity is dominated by resonant absorption, and is thus hardly understood as the potential energy of a quasiparticle. We rather introduced purely phenomenological pion potentials and fixed their value by analyzing pion production, pion absorption and proton production in pion-induced reactions at the same time. We found that, inside the nucleus, negative pions experience an attractive field of $-25 \mathrm{MeV}$ whereas positive pions feel a basically vanishing field. Of course, we refer here to the total (Coulomb + nuclear) field. Outside the nucleus, pions feel the Coulomb field. It turns out that the introduction of pion potentials is not sufficient to yield satisfactory results. We then investigated possible modifications of the pion production scenario in INCL4. We found that lowering the mass of $\Delta$-particles (by $\sim 50 \mathrm{MeV}$ ), as expected from medium effects [16], sometimes improves the results. The kind of results we then obtained is indicated in Figs. 1 and 2. Actually, agreement with experimental data is even better for light targets. Compared to our previous results [3], the production of pions is reduced, basically because of the introduction of the potentials, which hinders the decay of low-mass $\Delta$-particles.

\subsection{Improvements at low energy}

Theoretically, the INC model is valid above $\sim 250 \mathrm{MeV}$ only, a necessary condition for the separation of binary collisions in space and time. The practical validity of INCL has been investigated in detail recently [19]: it appears that this model gives reasonable results down to $\sim 50 \mathrm{MeV}$, for inclusive nucleon cross-sections, provided: (i) the experimental reaction cross-section is taken; (ii) the statistical Pauli blocking (collisions are accepted or rejected according to the phase space occupancy in the final state) is made 


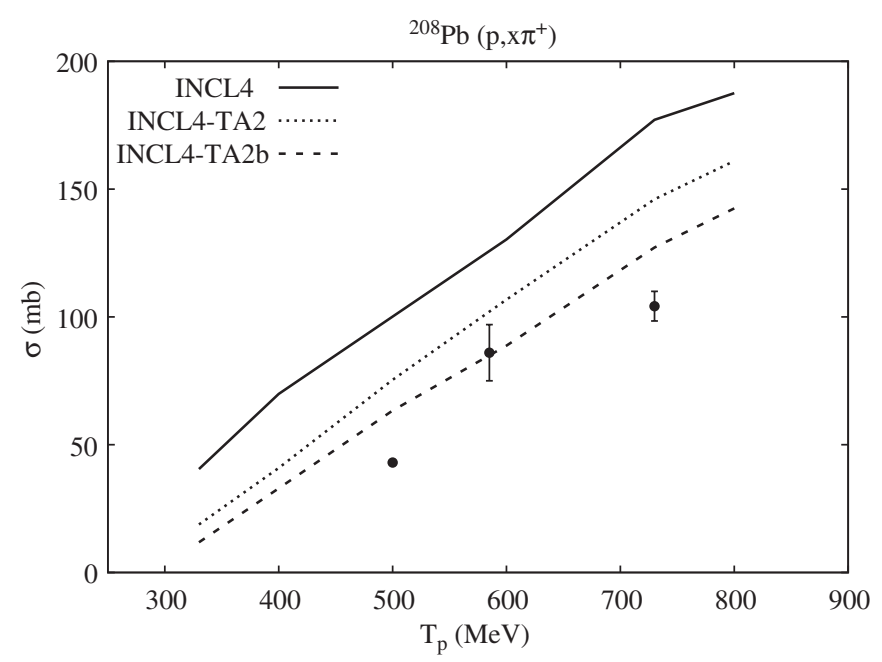

Fig. 1. Production of positive pions on ${ }^{208} \mathrm{~Pb}$. Results of INCL4 model (full curve), of INCL4 after introduction of phenomenological potentials for nucleons and pions (dotted curve), as explained in the text, and after modification of the $\Delta$-mass (dashed curve), with the experimental data (black dots) of Ref. [15].

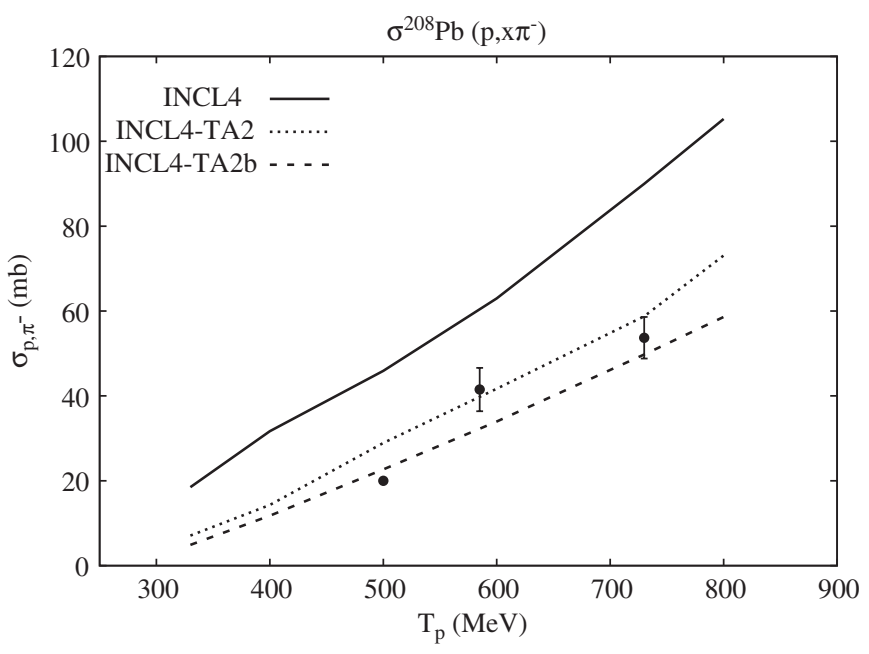

Fig. 2. Same as Fig. 1 for the production of negative pions on ${ }^{208} \mathrm{~Pb}$.

more strict. Actually, the best results seem to correspond to an implementation of the Pauli blocking which lies between the statistical procedure of INCL and the strict formulation. The statistical implementation of the Pauli blocking, accounting for a correct treatment when the Fermi sea is substantially depleted, is less and less appropriate at lower and lower energies. A strict implementation (collisions are rejected when final momenta are below the Fermi momentum) is obviously better founded at very low energy. See Ref. [19] for more details. A typical result is shown in Fig. 3. One can see that INCL4 yields reasonable agreement with experiment and competes relatively well with the sophisticated multi-step direct (MSD) calculation

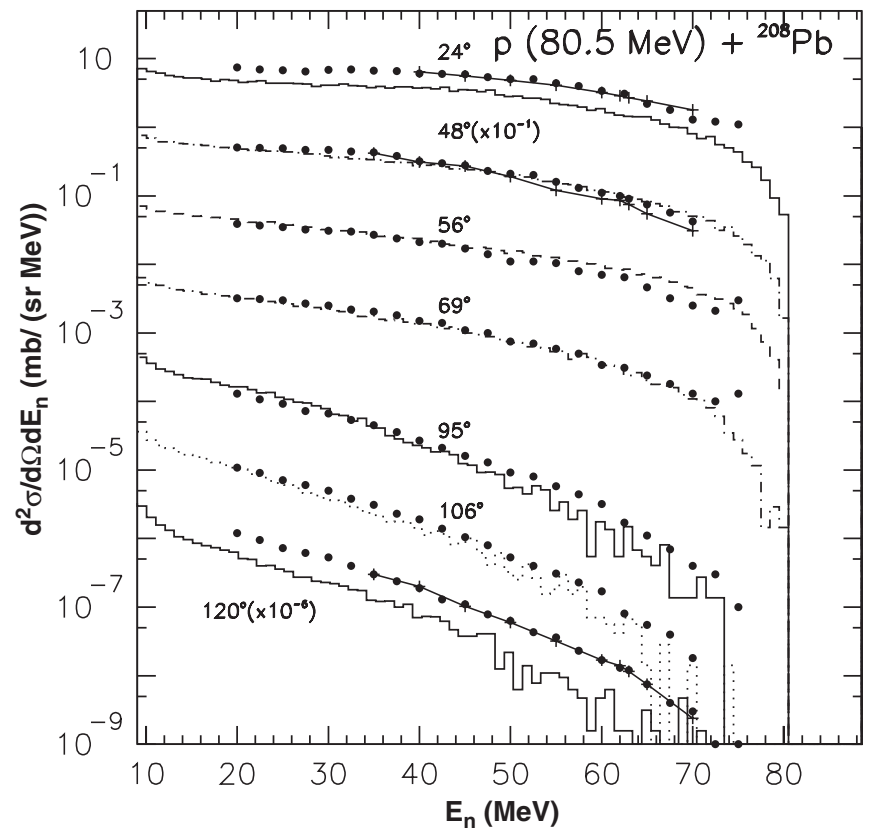

Fig. 3. Double differential neutron cross-section for $p+{ }^{208} \mathrm{~Pb}$ reactions at 80.5 MeV. Data (dots), from Ref. [17] are compared with the predictions of the INCL model (histograms) and with the multi-step direct (MSD) calculations of Ref. [18] (continuous lines). Adapted from Ref. [20].

of Ref. [18]. One has to realize that both models (MSD or ours) carry basically the same physics (multiple collisions in a degenerate Fermi gas), but with a different description of single-particle phase space (continuous in INCL4, discrete for MSD). In addition, MSD calculations introduce a certain number of adjustable parameters for the coupling to continuum, whereas, in INCL4, collisions and emission of nucleons are covered by the same and parameter-free model.

\section{Conclusion}

We have presented a few improvements of the INCL4 model, that have been worked out since the end of the HINDAS collaboration. They will be included in the next version of the INCL numerical code in the near future. They have to be paralleled by a similar effort to improve the de-excitation codes, as discussed in another contribution to this meeting [21].

\section{References}

[1] J.-J. Gaimard, K.-H. Schmidt, Nucl. Phys. A 531 (1991) 709.

[2] A.R. Junghans, et al., Nucl. Phys. A 629 (1998) 635.

[3] A. Boudard, J. Cugnon, S. Leray, C. Volant, Phys. Rev. C 66 (2002) 044615.

[4] M. Bernas, et al., Nucl. Phys. A 725 (2003) 213.

[5] C. Villagrasa, Ph.D. Thesis, Paris-Sud University, 2003.

[6] A. Boudard, J. Cugnon, J.-C. David, L. Donadille, S. Leray, C. Volant, in: Proceedings of the 6th International Conference on 
Nuclear Applications of Accelerator Technology, American Nuclear Society, Lagrange Park, USA, 2004. p. 339.

[7] C. Villagrasa, et al. in: R.C. Haight et al., (Eds.), International Conference on Nuclear Data for Science and Technology, AIP Conf. Proc. 769 (2005) 842.

[8] I. Leya, et al., Nucl. Instr. and Meth. B 229 (2005) 1.

[9] J.-P. Meulders, et al., HINDAS EU Contract FIKW-CT-2000-00031, Final Report, to be published.

[10] A. Koning, et al., J. Nucl. Sci. Tech. 2 (Suppl.) (2002) 1161.

[11] S. Leray, et al., Phys. Rev. C 65 (2002) 044621.

[12] P.E. Hodgson, The Nucleon Optical Potential, World Scientific, Singapore, 1994.
[13] A. Boudard, J. Cugnon, S. Leray, C. Volant, Nucl. Phys. A 740 (2004) 195.

[14] T. Aoust, J. Cugnon, Eur. Phys. J. A 21 (2004) 79.

[15] D.R.F. Cochran, et al., Phys. Rev. D 6 (1972) 3085.

[16] B. ter Haar, R. Malfliet, Phys. Rev. C 36 (1987) 1611.

[17] M. Trabandt, et al., Phys. Rev. C 39 (1989) 452.

[18] R. Bonetti, D. Camnasio, L. Colli Milazzo, P.E. Hodgson, Phys. Rev. C 24 (1981) 71

[19] P. Henrotte, Ph.D. Thesis, University of Liège, 2005.

[20] J. Cugnon, P. Henrotte, Eur. Phys. J. A 16 (2003) 393.

[21] S. Leray, Nucl. Instr. and Meth. (2006), these proceedings. 